\title{
Finite Element Simulation of Discrete Media With Granular Structure
}

\author{
R. Dittrich, T. Schrefl, Member, IEEE, H. Forster, D. Suess, W. Scholz, J. Fidler, Member, IEEE, and \\ V. Tsiantos, Member, IEEE
}

\begin{abstract}
Discrete media show great potential for future ultrahigh density magnetic recording. A hybrid finite element/boundary element method is used to compare the magnetization reversal process in a perpendicular granular film, a patterned media, and a single magnetic island. The results show that the influence of magnetostatic interactions on the switching field is comparable with the spread of the nucleation field due to the dispersion of the magnetic easy axes. For CoCrPt, this value is about $75 \mathrm{kA} / \mathrm{m}$.
\end{abstract}

Index Terms-Boundary element method, finite element simulation, patterned granular media, treecode.

\section{INTRODUCTION}

$\mathbf{T}$ HE term discrete media is used to refer to media that consist of arrays of discrete elements, for example ion-beam patterned magnetic elements [1], each of which can store one bit of data. Ideally, the storage density is then equal to the surface density of the elements. In patterned media, each discrete element is exchange-isolated from other elements, but inside each element polycrystalline grains are strongly exchange-coupled, behaving more like a larger single magnetic grain. Nevertheless, the micromagnetic simulations presented in Section III show that a single island reverses incoherently by the expansion of a small reversed nucleus. Section II of this paper describes a novel algorithm to treat the magnetostatic interactions between the islands using a hierarchical method. Section III compares the hysteresis behavior of a single island with a continuous film and a patterned media. In addition, we analyze the effect of the magnetostatic interactions on the coercive fields and calculate the dynamic coercivity as a function of the Gilbert damping constant.

\section{MicRomagnetics}

\section{A. Method}

The dynamic response of a magnetic particle to an applied field follows from the Gilbert equation of motion [2]

$$
\frac{\partial \mathbf{J}}{\partial t}=-|\gamma| \mathbf{J} \times \mathbf{H}_{\mathrm{eff}}+\frac{\alpha}{J_{s}} \mathbf{J} \times \frac{\partial \mathbf{J}}{\partial t} .
$$

Manuscript received February 12, 2002; revised June 3, 2002. This work was supported by the Austrian Science Fund Y132PHY.

The authors are with the Vienna University of Technology, A-1040 Vienna, Austria (e-mail: rok.dittrich@tuwien.ac.at).

Digital Object Identifier 10.1109/TMAG.2002.802786.
The effective field is obtained from the variational derivate of the total Gibbs free energy

$$
\begin{aligned}
\mathbf{H}_{\text {eff }} & =-\frac{\delta E_{t}}{\delta \mathbf{J}} \\
& =\mathbf{H}_{\text {exchange }}+\mathbf{H}_{\text {Zeeman }}+\mathbf{H}_{\text {anisotropy }}+\mathbf{H}_{\text {strayfield }} .
\end{aligned}
$$

We apply the finite element method [3] and backward differentiation scheme [4] to discretize the partial differential equation (1). For the calculation of strayfield, $\mathbf{H}_{\text {strayfield }}$, a novel numerical method is used which is explained in the next section.

\section{B. Calculations of the Stray Field}

The stray field is obtained from a boundary value problem

$$
\Delta u=\frac{\nabla \cdot \mathbf{J}_{s}}{\mu_{0}} \text { and } \quad \mathbf{H}_{s}=-\nabla u .
$$

A hybrid finite-element/boundary element method [5] is used to treat the magnetostatic interactions between the islands and to apply the boundary condition $u=0$ at infinity. The advantage of this method is that no finite elements are needed outside of the magnetic particle. For the solution of (3), we split $u$ into two parts, $u=u_{1}+u_{2}$. The potential $u_{1}$ is 0 outside of the magnetic particles and the solution of the Poisson equation with the boundary condition $\partial u_{1} / \partial n=\mathbf{J} \cdot \mathbf{n} / \mu_{0}$. Then the potential $u_{2}$ is solution of the Laplace equation with the boundary condition [6]

$$
\begin{array}{r}
u_{2}\left(\mathbf{x} \in \Gamma_{y}\right)=\frac{1}{4 \pi} \oiint_{\Gamma_{y}} \frac{u_{1}(\mathbf{y}) \cdot(\mathbf{x}-\mathbf{y})}{|\mathbf{x}-\mathbf{y}|^{3}} \mathbf{n} d \Gamma_{y} \\
+\left(\frac{\Omega(\mathbf{x})}{4 \pi}-1\right) u_{1}(\mathbf{x}) .
\end{array}
$$

Here, $\Gamma_{y}$ is the surface of the magnetic particles, and $\Omega$ is the solid angle. The direct evaluation of (4) requires a matrix vector product with a fully populated $N_{s} \times N_{s}$ matrix. Especially for thin films as in the case of patterned media, the number of surface nodes $N_{s}$ can get very high, since most nodes are located at the boundaries. The following method is more efficient. The first term of the right-hand side of (4) is the potential of a dipole sheet with the dipole density $u_{1} \mathbf{n}$ [6]. Therefore, the surface integral over the surface $\Gamma_{y}$ can be approximated by a sum over 


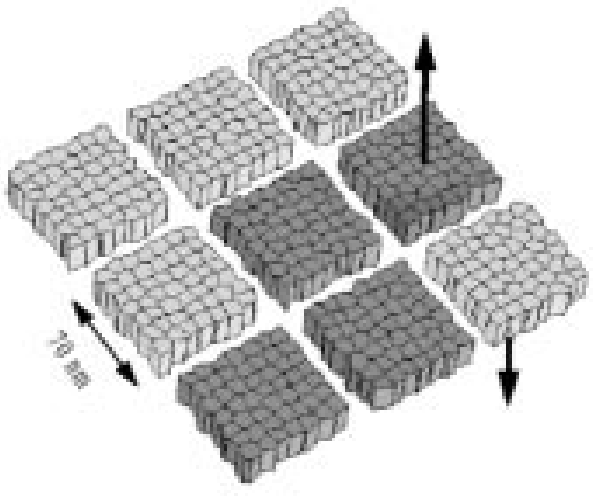

Fig. 1. Islands of the discrete media at an external field of $-870 \mathrm{kA} / \mathrm{m}$. Bright: Magnetization down. Dark: Magnetization up.

dipoles. The sum can be effectively evaluated using a tree code [7]. The potential $u_{2}$ at node $k$ is

$$
u_{2}^{k}=\sum_{i=1}^{N_{\Delta}} f\left(\mathbf{p}_{i}\right) .
$$

$N_{\Delta}$ is the number of surface triangles. Each surface triangle $\Delta_{k}$ has an assigned dipole $\mathbf{p}_{i}$ equal to the integral of the dipole density, $u_{1} \mathbf{n}$, over the triangle $i$. Since the dipole field decreases rapidly with the distance, dipoles far away from the node $k$ are grouped together forming one larger dipole. This method reduces the computational effort from $O\left(N_{S}^{2}\right)$ to $O\left(N_{S} \log N_{\Delta}\right)$. The second term on the right hand side of (4) is local and thus of order $O\left(N_{S}\right)$.

\section{RESULTS}

Based on the experimental work by IBM [1] we start with a continuous CoCrPt film (uniaxial anisotropy $K_{u}=0.3 \mathrm{MJ} / \mathrm{m}^{3}$, exchange constant $A=10 \mathrm{pJ} / \mathrm{m}$, magnetic polarization $J_{s}=$ $0.5 \mathrm{~T}$ ) consisting of 625 columnar grains, which are obtained from a Voronoi tessellation [8]. The grain diameter is $10 \mathrm{~nm}$ and the film thickness is $21 \mathrm{~nm}$. The easy axis is perpendicular to the film plane with a random deviation of the direction from the plane normal within a cone of about $8^{\circ}-10^{\circ}$ for each grain. In a second step, we take out elements in a grid pattern, to obtain an array of individual islands. The island size is $70 \mathrm{~nm}$ with a gap of $20 \mathrm{~nm}$ (Fig. 1).

\section{A. Hysteresis}

The subsequent calculation of equilibrium states solving the Gilbert equation of motion provides the demagnetization curve.

Fig. 1 shows a stable configuration at an applied field of $-870 \mathrm{kA} / \mathrm{m}$. The individual islands switch at different values of the external field. In contrast, the continuous film shows a single switching field (Fig. 2). In the continuous film, the magnetization starts to reverse near the center of one edge, forming a bubble-like domain. The domain expands leading to the reversal of the entire film. In a single island, the reversed domain is formed near one corner. The local demagnetizing field and the misorientation of the grains determine the nucleation field. The continuous film has a larger demagnetizing field and thus shows a lower switching field as compared to the single

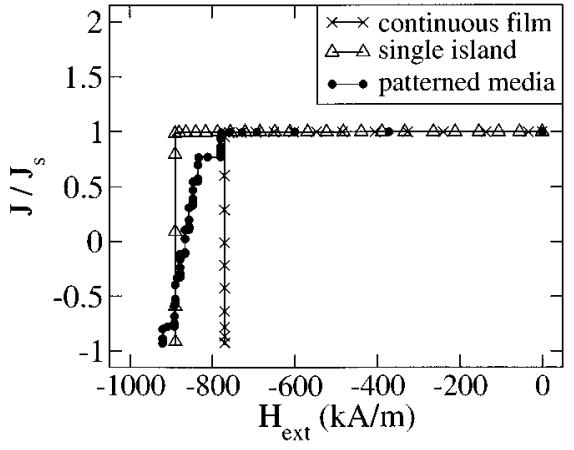

Fig. 2. Calculated demagnetization curves for the granular film, patterned media, and a single island.

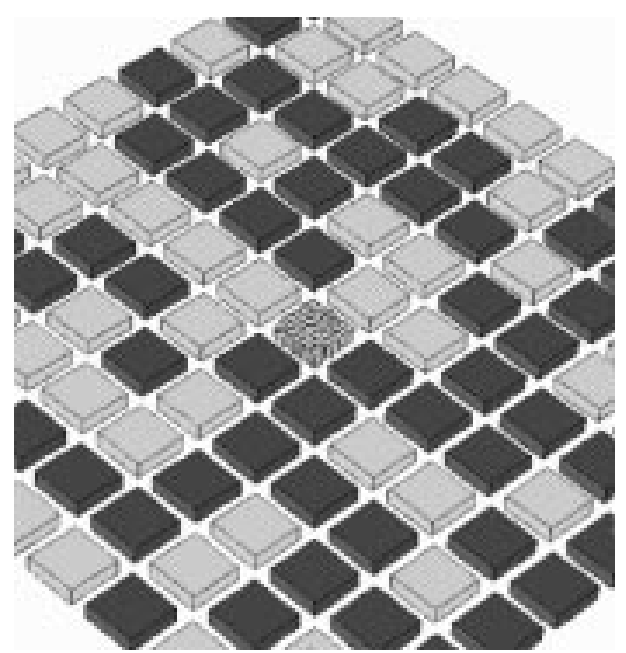

Fig. 3. Array of 121 islands as a model for patterned media. The magnetization is set random, representing the written state of a hard disk.

island. The granular film and the granular single island switch at a single switching field. The demagnetization curve of the patterned media shows steps at the switching fields of the individual islands. The spread of the coercive fields is $\Delta H_{c}=$ $144 \mathrm{kA} / \mathrm{m}$. In a reference calculation without a demagnetizing field, the spread in the switching field of the individual island is smaller $\left(\Delta H_{c}=84\right)$. The fields are about $230 \mathrm{kA} / \mathrm{m}$ higher than in a calculation with magnetostatic effects. This result suggests that the magnetostatic interactions increase the spread of the switching field.

\section{B. Dipolar Interactions}

To study the effect of the dipolar interactions, we considered an array of exchange decoupled islands, where each island represents one bit of data (Fig. 3). In order to reduce the computational effort, we simplify the model, focusing on the influence of dipolar interactions on the reversal process of a single island. We create an array of 121 islands. The "middle island" is the one of interest. It has granular structure consisting of 49 grains and is meshed with a fine grid of 5-nm finite-element size. This is fine enough to resolve domain walls for these certain material parameters (CoCr): $w=\pi \cdot \sqrt{A / K}=18 \mathrm{~nm}$.

The middle bit is surrounded with 120 additional islands. The only task of these islands is to produce the magnetic field. The 


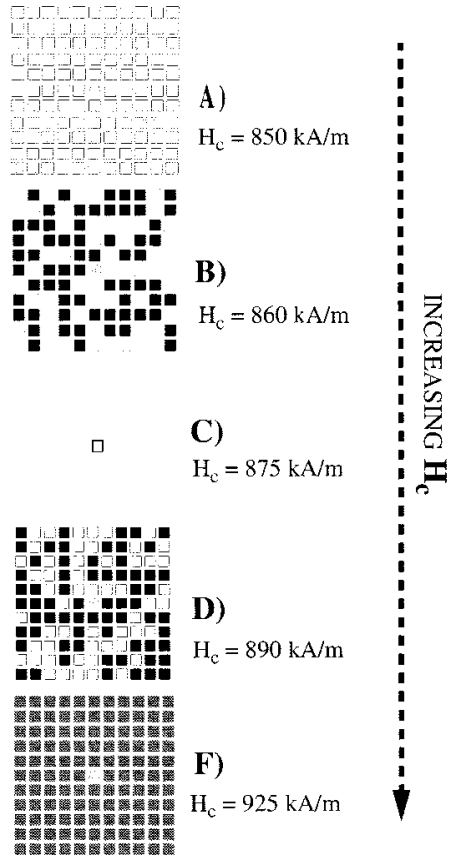

Fig. 4. Magnetizations states in patterned media. A) All neighbors "up"; B) random; C) isolated island without neighbors; D) random; and F) all neighbors "down."

external field is applied only in the region of the "middle island," which represents a very simple model of writing one bit on a patterned media. Since the magnetization of the "neighbor islands" is kept homogeneous and unchanged during the simulation a more coarse finite-element grid can be used for the surrounding islands. With increasing distance to the "middle island" the size of the finite elements is increased, since their influence on the "middle island" position decreases rapidly with the distance.

The magnetization state of Fig. 3 represents the bit pattern of a hard disk with written data. The written data (information) is assumed to look like a random magnetization pattern. For the average of many bits, the total magnetization should be zero. The hysteresis was calculated for several bit patterns (different data stored) in order to study the dispersion of the coercivity. The "worst case" is shown in Fig. 4(a) where all neighbors are magnetized in same direction as the middle bit leading to a demagnetizing field which favors the reversal of the middle bit. Therefore, in this case, the lowest coercivity is obtained while the "best case" (all neighbors are magnetized in opposite direction as the middle bit [see Fig. 4(f)] has the highest $H_{c}$ due to the stabilizing demagnetization field). In between, the more general cases with random magnetization states are found (B, D).

For data-storage applications, a unique dispersion of $H_{c}$ is desired since it enables the optimization of the write head and the switching time. The previous investigations show that dipolar interactions cause a maximum spread of $H_{c}$ of $75 \mathrm{kA} / \mathrm{m}$. This value is comparable to the dispersion of $H_{c}$ caused by the misorientation of grains.

\section{Writing Speed}

Beside the effort of reaching higher and higher area densities, another crucial point for magnetic recording applications

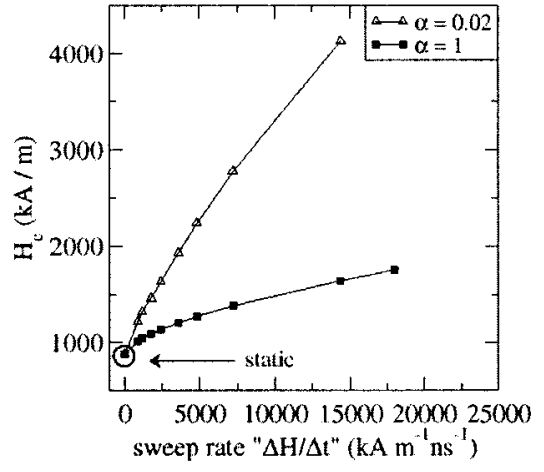

Fig. 5. Dynamical coercivity as a function of the field sweep rate for two different values of the damping constant $\alpha$.

is a fast writing speed in order to guarantee high data rates. To study the writing times of a single bit, an external field is applied perpendicular to the film plane which is increased linearly in time with different speeds (field "sweep rate"). Now $H_{c}$ will depend on the speed of change of the external field [9].

Calculations were made for low damping $(\alpha=0.02)$ and for high damping $(\alpha=1)$. Fig. 5 shows the calculated dynamical coercivity of the patterned media. $H_{c}$ depends both on the damping constant $\alpha$ and on the sweep rate of the external field. $H_{c}$ increases with increasing sweep rate of the external field. Thus a higher field has to be applied for higher writing speeds. This effect itself also depends on alpha and is stronger for smaller damping constants (Fig. 5).

For a very low sweeping rate of the external field, both for $\alpha=1$ and for $\alpha=0.02$, the $H_{c}$ approaches the limit obtained in a static hysteresis curve. This limit does not depend on the damping constant $\alpha$.

\section{ACKNOWLEDGMENT}

The authors would like to thank S. Anders and B. D. Terris, IBM Almaden Research Center, San Jose, CA, for helpful discussions.

\section{REFERENCES}

[1] C. T. Rettner, M. E. Best, and B. D. Terris, "Patterning of granular magnetic media with a focused ion beam to produce single-domain islands at > $140 \mathrm{Gbit} / \mathrm{in}^{2}$," IEEE Trans. Magn., vol. 37, pp. 1649-1651, July 2001.

[2] T. L. Gilbert, "A Lagrangian formulation of gyromagnetic equation of the magnetization field," Phys. Rev., vol. 100, p. 1243, 1955.

[3] T. Schrefl, J. Fidler, R. W. Chantrell, and M. Wongsam, "Micromagnetics II: Finite element approaches," in Encyclopedia of Materials: Science and Technology, K. H. J. Buschow, R. W. Cahn, M. C. Flemings, B. Ilschner, E. J. Kramer, and S. Mahajan, Eds. New York: Elsevier, 2001, pp. 5651-5661.

[4] S. D. Cohen and A. C. Hindmarsh, "CVODE, a stiff/nonstiff ODE solver in C," Comput. Phys., vol. 10, pp. 138-143, 1996.

[5] D. R. Fredkin and T. R. Koehler, "Hybrid method for computing demagnetizing fields," IEEE Trans. Magn., vol. 26, pp. 415-417, Jan. 1990.

[6] J. D. Jackson, Classical Electrodynamics, 2nd ed. New York: Wiley, 1975.

[7] J. Barnes and P. Hut, "A hierarchical $O(N \log N)$ force calculation algorithm," in Nature, 1986.

[8] T. Schrefl and J. Fidler, "Numerical simulation of magnetization reversal in hard magnets using a finite element method," J. Magn. Magn. Mater., vol. 111, pp. 105-114, 1992.

[9] D. Weller and A. Moser, "Thermal effect limits in ultrahigh-density magnetic recording," IEEE Trans. Magn., vol. 35, pp. 4423-4439, Nov. 1999. 